\title{
Progression after Immunotherapy for Fibrolamellar Carcinoma
}

\author{
Ulrike Bauer $^{\mathrm{a}}$ Carolin Mogler $^{\mathrm{b}} \quad$ Rickmer F. Braren $^{\mathrm{c}}$ Hana Algül ${ }^{\mathrm{a}}$ \\ Roland M. Schmid ${ }^{\mathrm{a}}$ Ursula Ehmer ${ }^{\mathrm{b}}$ \\ ${ }^{a}$ Department of Internal Medicine II, Klinikum rechts der Isar der Technischen Universität München, \\ Munich, Germany; ${ }^{b}$ Institute of Pathology, Technische Universität München, Munich, Germany; \\ 'Institute for Diagnostic and Interventional Radiology, Klinikum rechts der Isar der Technischen \\ Universität München, Munich, Germany
}

Keywords

Fibrolamellar carcinoma $\cdot$ Hepatocellular carcinoma · Immunotherapy · Checkpoint inhibitors

\section{Abstract}

Background: Fibrolamellar carcinoma (FLC) is a rare malignancy of the liver that differs from typical hepatocellular carcinoma (HCC) in several aspects such as the absence of underlying liver disease and occurrence in younger patients. Even though the survival rates in FLC are slightly better than in typical HCC, the prognosis of metastatic FLC remains deleterious. Several reports suggest that systemic chemotherapy regimens can successfully be used to halt disease progression in FLC, while targeted tumor therapy with sorafenib seems to be of limited efficiency. However, results from controlled clinical trials investigating systemic therapies in FLC are virtually nonexistent. Therefore, the choice of treatment often relies on case series with limited numbers of patients. Immunotherapy with checkpoint inhibitors is an emerging cancer therapy in several solid malignancies including HCC. Currently, there do not exist any reports on the use of checkpoint inhibitors in FLC. Case Report: Here, we describe a case of advanced FLC in a young man receiving immunotherapy, who progressed after 3 months of treatment - similar to 2 other patients with advanced FLC at our hospital. Conclusion: While immunotherapy seems to be a promising treatment with limited side effects in several other tumor entities, there is currently no data supporting tumor response in FLC. (c) 2019 S. Karger AG, Basel

\section{KARGER}

E-Mail karger@karger.com www.karger.com/vis

\section{Introduction}

Fibrolamellar carcinoma (FLC) is a rare subtype of hepatocellular carcinoma (HCC). However, the epidemiology and etiology of FLC differs substantially from typical HCC as the majority of FLC cases are diagnosed in younger patients ( $<40$ years of age) and are not associated with underlying liver disease. Additionally, recent studies indicate that the biology of FLC differs from typical HCC [1-3] and a DNAJB1-PRKACA fusion transcript has been identified as the signature genetic event in the tumor development of FLC $[2,4]$. While several studies indicate that the 5-year survival of patients with FLC (34-70\%) is better than for typical HCC (10-16\%) [5-7], this difference seems to be mainly attributable to the absence of cirrhosis in most FLC cases [8, 9].

Surgical resection is the primary treatment for FLC whenever possible and is associated with relatively good long-term survival even though recurrence rates of up to $90 \%$ remain extraordinarily high $[3,9]$. In unresectable hepatic tumors, transplantation remains a curative option with survival rates comparable to patients transplanted for HCC in more recent case series [10]. Advanced-stage tumors account for up to $20-30 \%$ of all FLC cases. Locally advanced tumor growth or systemic metastases both present limits for potentially curative treatments options such as liver transplantation or radiofrequency ablation. Therefore, the prognosis in advancedstage FLC tumors remains poor with less than $10 \%$ of patients surviving longer than 5 years $[5,6]$. Treatment in 


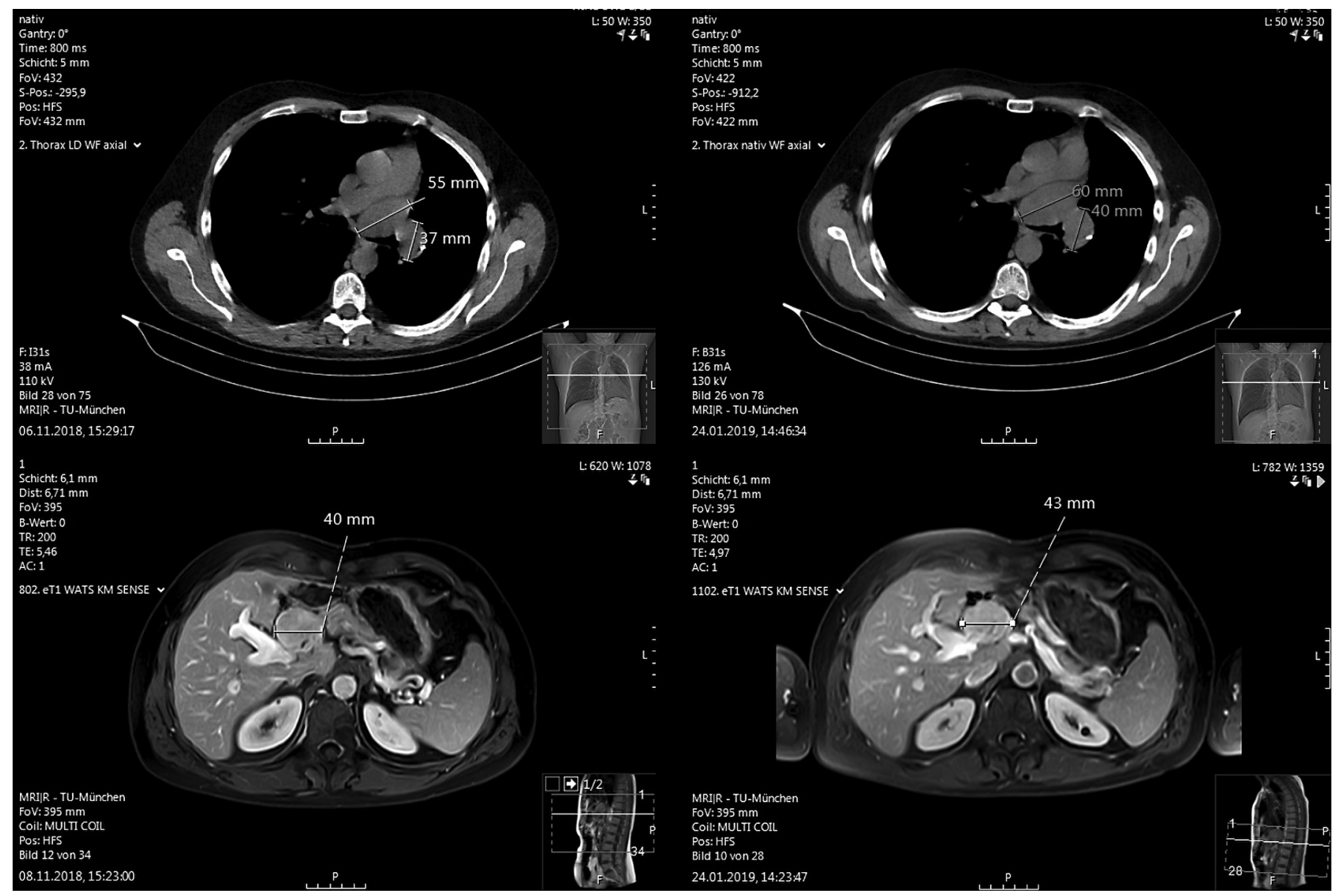

Fig. 1. Mediastinal lymph node metastasis (top) and liver tumor (bottom) before (left) and after (right) treatment with pembrolizumab.

these cases presents a challenge and no common guidelines or recommendations for the treatment of advanced FLC exist. Other than in typical HCC, systemic chemotherapy seems to be an efficient treatment option in some FLC patients [11-13]. However, the prognosis in patients treated with chemotherapy alone remains poor with a median survival of 20.6 months [12]. Novel targeted therapies such as sorafenib that significantly prolong overall survival in HCC have been used in the treatment of FLC. However, disease progression after 2.5-7 months of treatment reported in a small case series with 10 patients indicates that sorafenib might be of limited efficiency in FLC [13]. Therapy with tyrosine kinase inhibitors therefore remains controversial in FLC and efficient tumor therapies are urgently sought after. Checkpoint inhibitors present a novel class of systemic cancer therapeutics that trigger the activation of tumor-specific immunity. Immunotherapy with checkpoint inhibitors now plays a major role in modern oncologic treatment strategies. Via modulation of regulatory T-cell answers, they revoke suppression of tumor-specific immunoreactivity associated with an enhanced immunoreaction against tumor cells [14]. Phase II studies indicate that antibodies against PD-L1 - the ligand for the inhibitory checkpoint molecule PD-1 are of reasonable efficiency in advanced HCC $[15,16]$. However, it remains unknown to date whether FLC is responsive to immunotherapy. Here, we report a case of a patient with metastatic FLC who progressed on immunotherapy with pembrolizumab.

\section{Case Presentation}

We report on a 29-year-old male with a large tumor of the left hepatic lobe discovered incidentally by abdominal ultrasound. The patient did not suffer from any abdominal symptoms or other specific complaints and there were no abnormal laboratory findings. Liver enzymes and alpha-fetoprotein were within the normal rage. MRI scan confirmed tumor growth in the left liver lobe and a tumor biopsy showed a moderately differentiated FLC. Sequence analysis of tumor tissue performed later revealed the presence of the DNAJB1-PRKACA gene fusion characteristic of FLC.

The patient underwent surgery (hemihepatectomy of left lobe segment II-III) and follow-up resection (lymphadenectomy, liver segment IV). Due to abdominal metastasis further surgery was necessary 2 years later (resection of diaphragm, parts of jejunum, atypical resection of the superior lobe of the left lung).

Yet another 2 years later, the patient presented with partial loss of vision. MRI and CT scans revealed cerebral metastasis to the 


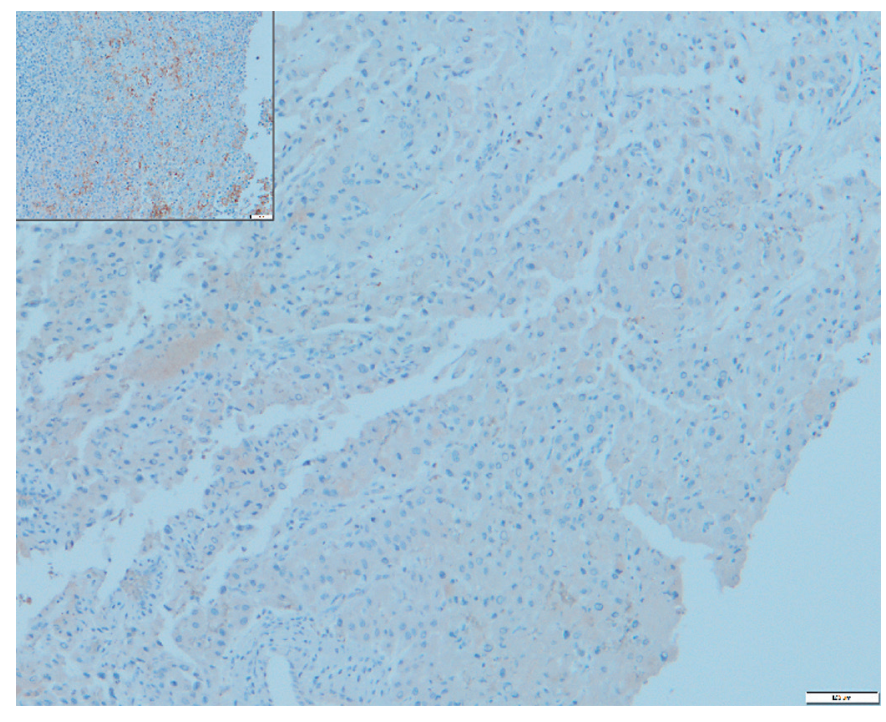

Fig. 2. Immunostaining of cerebral metastasis of fibrolamellar carcinoma for PD-L1. Inset Positive control.

sellar region, mediastinal lymph node metastases, and a recurrent liver lesion. The patient was then referred to our clinic for further treatment. Two subsequent R1 resections of cerebral metastasis were performed, followed by stereotactic radiation of the sellar region resulting in major improvement of the patients' vision.

With the diagnosis of a nonresectable FLC we initiated systemic tumor therapy with sorafenib. The medication was not well tolerated, with the need of repeated discontinuation and dose reduction due to treatment-related side effects, mainly hand-foot syndrome. Routine staging MRI and CT scans revealed progression of the hepatic tumor as well as of mediastinal metastases 3 months after start of treatment with sorafenib. Therefore, treatment with sorafenib was discontinued. The patient then received immunotherapy with pembrolizumab for 2.5 months $(200 \mathrm{mg}$ intravenously every 3 weeks). The treatment was well tolerated with no apparent side effects. However, after 2.5 months of therapy MRI of the liver and CT scan of the chest again showed progressive disease (Fig. 1).

\section{Discussion}

FLC is a rare primary cancer of the liver that mainly occurs in younger patients with no underlying liver disease. In advanced stages, prognosis is very poor with a median survival of 12 months and only minor advances in FLC-specific systemic therapies in recent years [6, 17]. As there are only few patients with this rare disease, controlled studies regarding the treatment of advanced FLC are limited and therapeutic approaches are therefore either adapted from typical HCC or based on small treatment series or case reports. There are no specific recommendations or guidelines. While several studies show a benefit of different systemic chemotherapies, these reports are based on small case series and have shown only limited long-term efficiency [17]. Targeted tumor therapies have not been investigated in FLC and a retrospective analysis of 10 patients receiving sorafenib indicated limited efficiency [13]. However, recent advances in the molecular characterization of FLC revealed potential targets including the mTOR pathway or Aurora A kinase. However, even with favorable outcome of mTOR inhibition in single patients [18], no promising results have been published from controlled trials to date [4].

Treatment with checkpoint inhibitors is established in several solid malignancies including melanoma, lung cancer, renal cell carcinoma, and head-and-neck cancer [19] and seems to be a promising therapeutic approach in HCC $[15,16]$. Several tumor characteristics seem to favor a response towards checkpoint inhibitors: tumor-inherent genomic instability and high mutational load are associated with an improved overall survival $[19,20]$. While FLC is generally characterized by a low mutational load in comparison to other cancers, next-generation sequencing of tumor tissue revealed the presence of $\mathrm{MSH} 2$ mutations with a frequency of $41 \%$ as a marker of genomic instability in our patient. Furthermore, there is evidence that radiation therapy induces a synergistic anti-tumor response in combination with checkpoint inhibitors through multiple effects on cancer cells and on the tumor microenvironment [21]. Stereotactic irradiation of cerebral metastasis and the presence of a marker for genomic instability therefore seemed be predictors of a favorable response to immunotherapy in our patient. On the other hand, immunostaining of metastatic tumor tissue did not show any PD-L1 expression (Fig. 2), which has been associated with poor tumor response in several malignancies, but not in HCC.

While progression after 2.5 months of treatment with pembrolizumab was below $20 \%$, after 3 months of treatment in our patient the increase in tumor size was comparable to previous progression under sorafenib and the low growth rate during the earlier course of the disease. These findings strongly indicate that treatment with PD-L1 antibodies is likely not beneficial in our patient.

At our clinic, 2 more patients with advanced FLC and lung metastases have been treated with the PD-L1 inhibitor nivolumab in recent years. Both developed progressive disease after 2 and 3 months, respectively. Interestingly, one patient had received selective internal radiotherapy (SIRT) for her large hepatic tumor mass and displayed markers of genomic instability in sequenced tumor tissue. Those predictive markers - radiation therapy and genomic instability - were again not associated with any tumor response in our patient.

While a report of a total of 3 cases is not sufficient to deduct any therapeutic recommendations against immunotherapy in FLC, it might be worth considering other 
options such as systemic chemotherapy before switching to an experimental therapy with no positive case reports published to date. Nevertheless, immunotherapy in FLC might be just as efficient as in HCC where objective response rates are below $30 \%$ in two phase II trials. Additionally, the significance of PD-L1 expression as a potential predictor for tumor response in FLC needs to be evaluated.

In summary, there is no current evidence for a response upon treatment with checkpoint inhibitors in FLC and further studies will be needed to achieve improvement in the outcome of advanced FLC.

\section{Statement of Ethics}

Written informed consent to publish this case (including publication of images) was provided by the patient.

\section{Disclosure Statement}

The authors have no conflicts of interest to declare.

\section{Author Contributions}

U.B., H.A., R.M.S., and U.E. drafted and wrote the manuscript. C.M. prepared and analyzed the immunohistochemistry stainings. R.F.B. reviewed and prepared the radiology pictures.

\section{References}

1 Liu S, Chan KW, Wang B, Qiao L. Fibrolamellar hepatocellular carcinoma. Am J Gastroenterol. 2009 Oct;104(10):2617-24; quiz 2625.

2 Honeyman JN, Simon EP, Robine N, Chiaroni-Clarke R, Darcy DG, Lim II, et al. Detection of a recurrent DNAJB1-PRKACA chimeric transcript in fibrolamellar hepatocellular carcinoma. Science. 2014 Feb;343(6174): $1010-4$.

3 Malouf GG, Brugières L, Le Deley MC, Faivre S, Fabre M, Paradis V, et al. Pure and mixed fibrolamellar hepatocellular carcinomas differ in natural history and prognosis after complete surgical resection. Cancer. 2012 Oct; 118(20):4981-90

4 Lalazar G, Simon SM. Fibrolamellar Carcinoma: Recent Advances and Unresolved Questions on the Molecular Mechanisms. Semin Liver Dis. 2018 Feb;38(1):51-9.

5 Eggert T, McGlynn KA, Duffy A, Manns MP, Greten TF, Altekruse SF. Epidemiology of fibrolamellar hepatocellular carcinoma in the USA, 2000-10. Gut. 2013 Nov;62(11):1667-8.

6 Stipa F, Yoon SS, Liau KH, Fong Y, Jarnagin WR, D'Angelica M, et al. Outcome of patients with fibrolamellar hepatocellular carcinoma. Cancer. 2006 Mar;106(6):1331-8.

7 El-Serag HB. Hepatocellular carcinoma. N Engl J Med. 2011 Sep;365(12):1118-27.

8 Njei B. Fibrolamellar hepatocellular carcinoma versus conventional hepatocellular carcinoma: better 5-year survival or artifactual result of research methodology? Gut. 2014 Sep; 63(9):1374-5.
9 Yamashita S, Vauthey JN, Kaseb AO, Aloia TA, Conrad C, Hassan MM, et al. Prognosis of Fibrolamellar Carcinoma Compared to Non-cirrhotic Conventional Hepatocellular Carcinoma. J Gastrointest Surg. 2016 Oct; 20(10):1725-31

10 Atienza LG, Berger J, Mei X, Shah MB, Daily MF, Grigorian A, et al. Liver transplantation for fibrolamellar hepatocellular carcinoma: A national perspective. J Surg Oncol. 2017 Mar; 115(3):319-23.

11 Patt YZ, Hassan MM, Lozano RD, Brown TD, Vauthey JN, Curley SA, et al. Phase II trial of systemic continuous fluorouracil and subcutaneous recombinant interferon Alfa-2b for treatment of hepatocellular carcinoma. J Clin Oncol. 2003 Feb;21(3):421-7.

12 Kaseb AO, Shama M, Sahin IH, Nooka A, Hassabo HM, Vauthey JN, et al. Prognostic indicators and treatment outcome in 94 cases of fibrolamellar hepatocellular carcinoma. Oncology. 2013;85(4):197-203.

13 Ang CS, Kelley RK, Choti MA, Cosgrove DP, Chou JF, Klimstra D, et al. Clinicopathologic characteristics and survival outcomes of patients with fibrolamellar carcinoma: data from the fibrolamellar carcinoma consortium. Gastrointest Cancer Res. 2013 Jan;6(1): 3-9.

14 Pardoll DM. The blockade of immune checkpoints in cancer immunotherapy. Nat Rev Cancer. 2012 Mar;12(4):252-64.

15 El-Khoueiry AB, Sangro B, Yau T, Crocenzi TS, Kudo M, Hsu C, et al. Nivolumab in patients with advanced hepatocellular carcinoma (CheckMate 040): an open-label, noncomparative, phase $1 / 2$ dose escalation and expansion trial. Lancet. 2017 Jun;389(10088): 2492-502.
16 Zhu AX, Finn RS, Edeline J, Cattan S, Ogasawara S, Palmer D, et al.; KEYNOTE-224 investigators. Pembrolizumab in patients with advanced hepatocellular carcinoma previously treated with sorafenib (KEYNOTE-224): a non-randomised, open-label phase 2 trial. Lancet Oncol. 2018 Jul;19(7):940-52.

17 Kassahun WT. Contemporary management of fibrolamellar hepatocellular carcinoma: diagnosis, treatment, outcome, prognostic factors, and recent developments. World J Surg Oncol. 2016 May;14(1):151.

18 Bill R, Montani M, Blum B, Dufour JF, Escher $R$, Bühlmann M. Favorable response to mammalian target of rapamycin inhibition in a young patient with unresectable fibrolamellar carcinoma of the liver. Hepatology. 2018 Jul; 68(1):384-6.

19 Rotte A, Jin JY, Lemaire V. Mechanistic overview of immune checkpoints to support the rational design of their combinations in cancer immunotherapy. Ann Oncol. 2018 Jan; 29(1):71-83.

20 Yaghmour G, Pandey M, Ireland C, Patel K, Nunnery S, Powell D, et al. Role of Genomic Instability in Immunotherapy with Checkpoint Inhibitors. Anticancer Res. 2016 Aug; 36(8):4033-8.

21 Salama AK, Postow MA, Salama JK. Irradiation and immunotherapy: from concept to the clinic. Cancer. 2016 Jun;122(11):1659-71. 